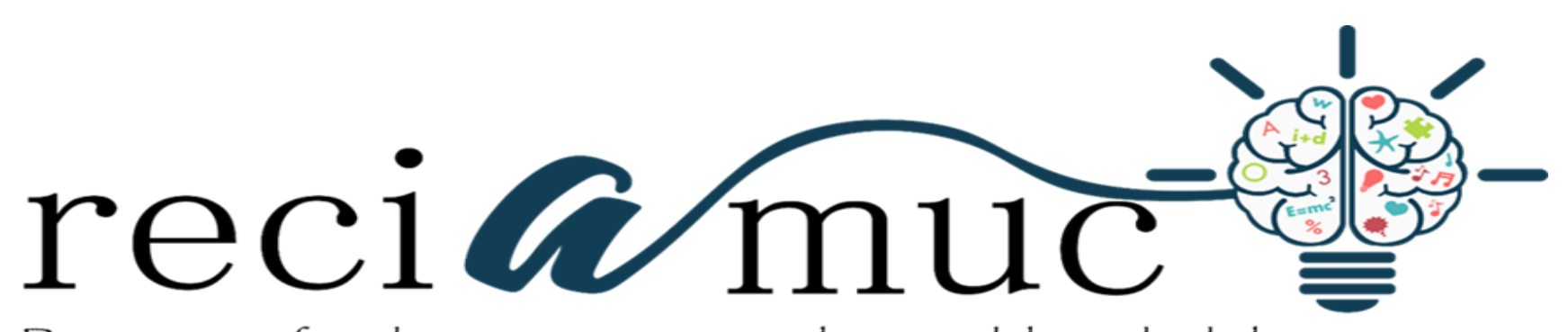

Revista científica de investigación actualización del mundo de las ciencias

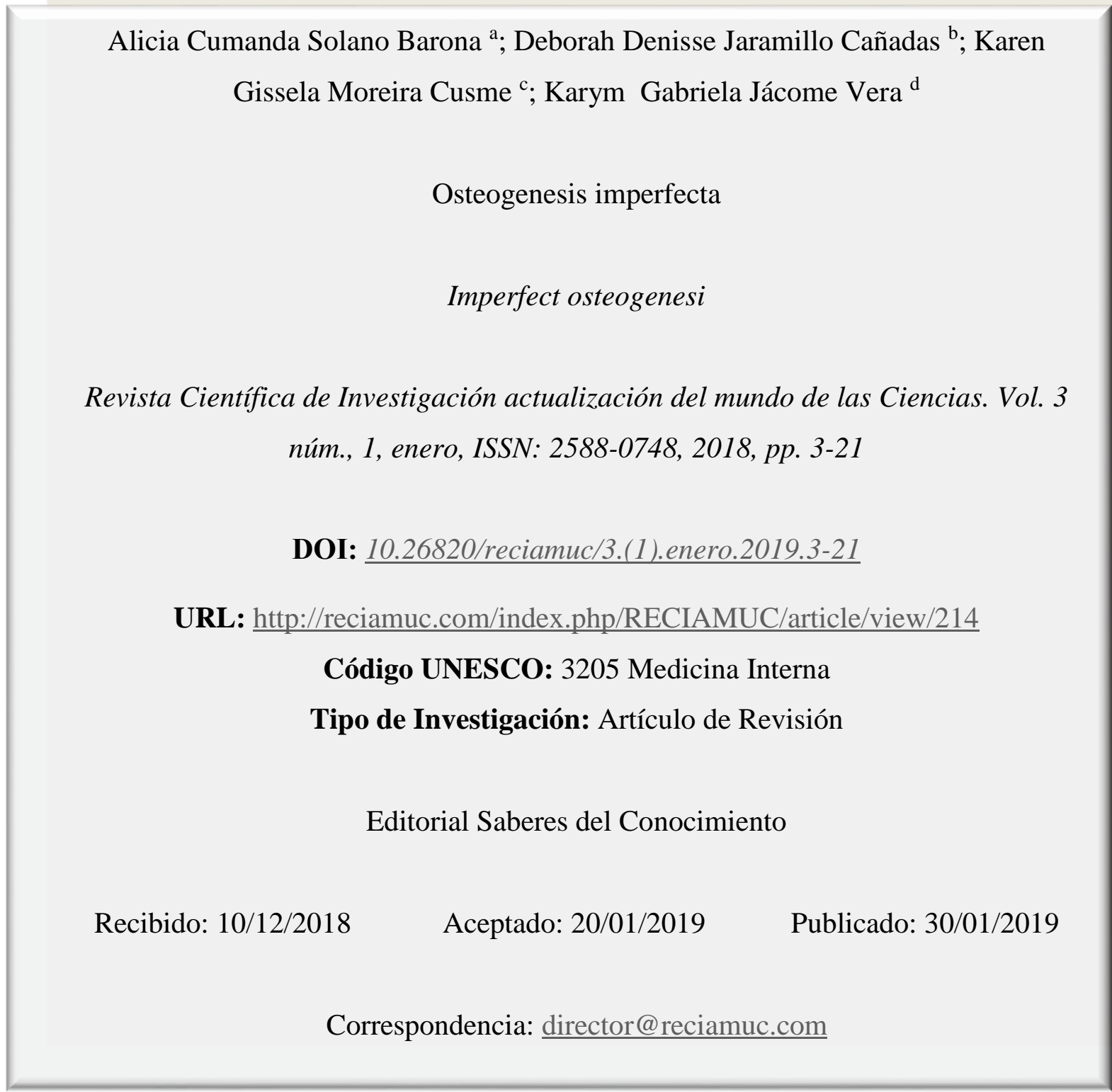
a. Médico; Guayaquil, Ecuador.
b. Médico; Guayaquil, Ecuador.
c. Médico; Guayaquil, Ecuador.
d. Médico; Guayaquil, Ecuador. 


\section{Osteogenesis imperfecta}

Vol. 3, núm. 1., (2019)

Alicia Cumanda Solano Barona; Deborah Denisse Jaramillo Cañadas; Karen Gissela Moreira Cusme; Karym Gabriela Jácome Vera

\section{RESUMEN}

La osteogénesis imperfecta (OI) es un trastorno heterogéneo raro del tejido conectivo comúnmente causado por mutaciones en el colágeno del gen tipo 1. Es un trastorno que se ve a nivel mundial, independientemente de la edad, el género o el grupo étnico y se presenta tanto en niños como en adultos. Las características clínicas típicas son hueso quebradizo, alta frecuencia de fracturas y deformidades óseas. Los signos observados son la esclerótica azul, la dentinogénesis imperfecta y la otosclerosis. En esta revisión, el autor hace una revisión sistemática. General, incluidos los mecanismos, la clasificación, los métodos de diagnóstico, relacionados con las enfermedades de interés humano y tratamiento. La revisión también se centra en la OI y su vinculación con muchas enfermedades relacionadas con la salud. Pacientes con OI deben tener un seguimiento médico de esta enfermedad de lo contrario, las deformidades óseas y los defectos del colágeno comunes a la OI pueden afectar varios órganos internos, dando lugar a problemas secundarios mayores o menores. La optimización del tratamiento en niños y adultos sigue siendo un desafío, ya que, los tratamientos disponibles no se dirigen al defecto de colágeno subyacente. El tratamiento empleado en este tipo de patología incluye fisioterapia, procedimientos quirúrgicos y farmacoterapia. En esta breve reseña, el autor discute principalmente. En esta breve reseña, el autor discute principalmente el conocimiento actual de las terapias farmacológicas y posibles terapias futuras para el tratamiento de la OI.

Palabras Claves: Colágeno Tipo 1; Osteogénesis Imperfecta; Bifosfonatos; Neridronato y Teriparatida. 


\title{
Osteogenesis imperfecta
}

Vol. 3, núm. 1., (2019)

Alicia Cumanda Solano Barona; Deborah Denisse Jaramillo Cañadas; Karen Gissela Moreira Cusme; Karym Gabriela Jácome Vera

\begin{abstract}
Osteogenesis imperfecta (OI) is a rare heterogeneous connective tissue disorder commonly caused by mutations in the collagen of the type 1 gene. It is a disorder that is seen worldwide, regardless of age, gender or ethnic group and is presented both in children and adults. The typical clinical features are brittle bone, high frequency of fractures and bone deformities. The signs observed are blue sclera, dentinogenesis imperfecta and otosclerosis. In this review, the author makes a systematic review. General, including mechanisms, classification, diagnostic methods, related to diseases of human interest and treatment. The review also focuses on OI and its link to many health-related diseases. Patients with OI must have a medical follow-up of this disease otherwise, bone deformities and collagen defects common to OI may affect several internal organs, leading to major or minor side problems. The optimization of treatment in children and adults remains a challenge, since the available treatments do not address the underlying collagen defect. The treatment used in this type of pathology includes physiotherapy, surgical procedures and pharmacotherapy. In this brief review, the author discusses mainly. In this brief review, the author discusses mainly the current knowledge of pharmacological therapies and possible future therapies for the treatment of OI.
\end{abstract}

Key Words: Collagen Type 1; Imperfect osteogenesis; Bisphosphonates; Neridronate and Teriparatide. 


\section{Osteogenesis imperfecta}

Vol. 3, núm. 1., (2019)

Alicia Cumanda Solano Barona; Deborah Denisse Jaramillo Cañadas; Karen Gissela Moreira Cusme; Karym Gabriela Jácome Vera

\section{Introducción.}

La osteogénesis imperfecta (OI) es un trastorno genético caracterizado por huesos que se rompen fácilmente, literalmente significa hueso imperfectamente formado. Las personas con OI tienen un defecto genético que afecta la capacidad del cuerpo para hacer huesos fuertes También se le llama como enfermedad de los huesos frágiles.

Los pacientes afectados tienen múltiples fracturas desde la infancia, tanto traumatismo espontáneo como fracturas relacionadas con un mínimo esfuerzo fisico. Los niños afectados suelen morir en el período neonatal. (Rauch F, 2010).

El autor (Zhao X, 2011) en su estudio expresa que:

La OI es una rara enfermedad clínica, que se presenta en una tasa de entre 1 / 10,000 y 1 / 25,000 en todo el mundo. La falta de buena comprensión de las dificultades en el diagnóstico, tratamiento óptimo, y avances recientes sobre la enfermedad han desencadenado un problema de salud mundial debido a errores en el diagnostico o diferencia de opiniones. (p. 22).

De acuerdo a la complejidad de esta patología, el autor se centra en la necesidad de realizar una revisión sistemática acerca de la OI, a través de la realización de este trabajo de investigación con el fin de comprender de mejor manera esta enfermedad y tener conocimiento para tratar a los pacientes que la padecen. 


\section{Osteogenesis imperfecta}

Vol. 3, núm. 1., (2019)

Alicia Cumanda Solano Barona; Deborah Denisse Jaramillo Cañadas; Karen Gissela Moreira Cusme; Karym Gabriela Jácome Vera

El autor (Joseph L S, 2015) define este esta patología como:

Una enfermedad hereditaria principalmente causada por un defecto de colágeno, debido a mutaciones en los genes que codifican las cadenas del gen colágeno tipo I, ocasionando generalmente a la OI autosómica dominante. El colágeno tipo I es una estructura en forma de varilla formada por un trímero de 2 COL1A1 y 1 COL1A2 subunidades, que requiere modificación post-traduccional. Muchas de las otras formas raras de OI se deben a defectos en las proteínas involucradas en reticulación, hidroxilación y mineralización del colágeno tipo I. (p. 13).

Otros estudios de biología molecular han encontrado que las dos cadenas han repetido estructuras de triplete dextro-girado de glicina-prolina-hidroxiprolina. La estructura del triplete es extremadamente importante para el plegado correcto de la cadena peptídica. Lo que presenta anormalidad en este triplete causada por genes, estos cambios, conducen a una alineación incorrecta del péptido y, finalmente, a la anomalía en el colágeno tipo 1. (Wagner, 2012).

Además, se cree que la proteína asociada al cartílago (CRTAP), proly 13-hidroxilasa 1 (P3H1 / LEPRE1) y ciclofilina B (CyPB/PPIB) también influyen en esta estructura de triplete, y pueden participar en la patogenia de la OI. "La principal manifestación clínica es la fragilidad esquelética. Puede haber deformidad esquelética, laxitud articular y esclerosis. Otro tipo de manifestaciones esqueléticas incluyen pérdida de audición, dentinogénesis imperfecta, esclerótica azul / gris, sordera progresiva, rebordeado de las costillas, hipercalciuria, dilatación de la raíz aórtica, complicaciones vasculares, pulmonares y neurológicas. Condiciones tales como macrocefalia, hidrocefalia e invaginación basilar” (Biggin A, 2014). 


\section{Osteogenesis imperfecta}

Vol. 3, núm. 1., (2019)

Alicia Cumanda Solano Barona; Deborah Denisse Jaramillo Cañadas; Karen Gissela Moreira Cusme; Karym Gabriela Jácome Vera

El fenotipo es variable, desde la osteoporosis que se presenta en la edad adulta hasta la letalidad en los niños. Incluso los adultos con OI "leve" pueden tener síntomas musculoesqueléticos significativos, que incluyen artritis, fracturas, espalda, dolor, escoliosis y roturas tendinosas.

\section{Métodos y materiales.}

Para el desarrollo de este proceso investigativo, se plantea como metodología la encaminada hacia una orientación científica particular que se encuentra determinada por la necesidad de indagar en forma precisa y coherente una situación, en tal sentido (Dávila., 2015) define la metodología "como aquellos pasos previos que son seleccionados por el investigador para lograr resultados favorables que le ayuden a plantear nuevas ideas".(p.66)

Lo citado por el autor, lleva a entender que el desarrollo de la acción investigativa busca simplemente coordinar acciones enmarcadas en una revisión bibliográfica con el fin de complementar ideas previas relacionado con la Osteogenesis Imperfecta, a través de una revisión de literatura, para así finalmente elaborar un cuerpo de consideraciones generales que ayuden a ampliar el interés propuesto.

\section{Tipo de Investigación}

Dentro de toda práctica investigativa, se precisan acciones de carácter metodológico mediante las cuales, se logra conocer y proyectar los eventos posibles que la determinan, así como las características que hacen del acto científico un proceso interactivo ajustado a una realidad posible de ser interpretada. En este sentido, se puede decir, que la presente investigación 


\section{Osteogenesis imperfecta}

Vol. 3, núm. 1., (2019)

Alicia Cumanda Solano Barona; Deborah Denisse Jaramillo Cañadas; Karen Gissela Moreira Cusme; Karym Gabriela Jácome Vera

corresponde al tipo documental, definido por (Castro, 2016), "se ocupa del estudio de problemas planteados a nivel teórico, la información requerida para abordarlos se encuentra básicamente en materiales impresos, audiovisuales y /o electrónicos”. (p.41).

En consideración a esta definición, la orientación metodológica permitió la oportunidad de cumplir con una serie de actividades inherentes a la revisión y lectura de diversos documentos donde se encontraron ideas explicitas relacionadas con los tópicos encargados de identificar a cada característica insertada en el estudio. Por lo tanto, se realizaron continuas interpretaciones con el claro propósito de revisar aquellas apreciaciones o investigaciones propuestas por diferentes investigadores relacionadas con el tema de interés, para luego dar la respectiva argumentación a los planteamientos, en función a las necesidades encontradas en la indagación.

\section{Fuentes Documentales}

El análisis correspondiente a las características que predomina en el tema seleccionado, llevan a incluir diferentes fuentes documentales encargadas de darle el respectivo apoyo y en ese sentido cumplir con la valoración de los hechos a fin de generar nuevos criterios que sirven de referencia a otros procesos investigativos. Para (Castro, 2016) las fuentes documentales incorporadas en la investigación documental o bibliográfica, "representa la suma de materiales sistemáticos que son revisados en forma rigurosa y profunda para llegar a un análisis del fenómeno". (p.41). Por lo tanto, se procedió a cumplir con la realización de una lectura previa determinada para encontrar aquellos aspectos estrechamente vinculados con el tema, con el fin de explicar mediante un desarrollo las respectivas apreciaciones generales de importancia.

\section{Técnicas para la Recolección de la Información}




\section{Osteogenesis imperfecta}

Vol. 3, núm. 1., (2019)

Alicia Cumanda Solano Barona; Deborah Denisse Jaramillo Cañadas; Karen Gissela Moreira Cusme; Karym Gabriela Jácome Vera

La conducción de la investigación para ser realizada en función a las particularidades que determinan a los estudios documentales, tiene como fin el desarrollo de un conjunto de acciones encargadas de llevar a la selección de técnicas estrechamente vinculadas con las características del estudio. En tal sentido, (Bolívar, 2015), refiere, que es "una técnica particular para aportar ayuda a los procedimientos de selección de las ideas primarias y secundarias". (p. 71).

Por ello, se procedió a la utilización del subrayado, resúmenes, fichaje, como parte básica para la revisión y selección de los documentos que presentan el contenido teórico. Es decir, que mediante la aplicación de estas técnicas se pudo llegar a recoger informaciones en cuanto a la revisión bibliográfica de los diversos elementos encargados de orientar el proceso de investigación. Tal como lo expresa, (Bolívar, 2015) "las técnicas documentales proporcionan las herramientas esenciales y determinantes para responder a los objetivos formulados y llegar a resultados efectivos" (p. 58). Es decir, para responder con eficiencia a las necesidades investigativas, se introdujeron como técnica de recolección el método inductivo, que hizo posible llevar a cabo una valoración de los hechos de forma particular para llegar a la explicación desde una visión general.

Asimismo, se emplearon las técnicas de análisis de información para la realización de la investigación que fue ejecutada bajo la dinámica de aplicar diversos elementos encargados de determinar el camino a recorrer por el estudio, según, (Bolívar, 2015) las técnicas de procesamiento de datos en los estudios documentales "son las encargadas de ofrecer al investigador la visión o pasos que debe cumplir durante su ejercicio, cada una de ellas debe estar en correspondencia con el nivel a emplear" (p. 123). Esto indica, que para llevar a cabo el procesamiento de los datos obtenidos una vez aplicado las técnicas seleccionadas, tales como: 


\section{Osteogenesis imperfecta}

Vol. 3, núm. 1., (2019)

Alicia Cumanda Solano Barona; Deborah Denisse Jaramillo Cañadas; Karen Gissela Moreira Cusme; Karym Gabriela Jácome Vera

fichas de resumen, textual, registros descriptivos entre otros, los mismos se deben ajustar al nivel que ha sido seleccionado.

\section{Resultados}

Sintomas y signos

Existen diferentes tipos de OI con síntomas que van desde leve a severa, cada persona con la condición puede tener una combinación diferente de síntomas. "Todas las personas con OI, tienen huesos más débiles, algunos síntomas comunes de la OI incluyen: Estatura corta, triangular, cara conformada, problemas respiratorios, pérdida de audición, dientes quebradizos, huesos de deformidades tales como piernas arqueadas de escoliosis". (Salvador, 2011)

Hay varios tipos de OI que varian de acuerdo a la severidad del caso y las características estudios hanrevelado nuevos problemas genéticos que ocasionan la OI incluyendo trastornos y síntomas involucrados y que se describen a continuación:

\section{Clasificación}

El autor (Sillence DO, 1979) dividió la enfermedad en cuatro tipos del I al IV. La mayoría de los casos de OI (posiblemente del 85 al 90 por ciento) son causados por una mutación dominante en un gen que codifica colágeno tipo 1. Esta clasificación se mantuvo durante mucho tiempo, sin embargo, hasta hace poco, un estudio realizado por (Starr, 2010) reveló la existencia más tipos de OI. A continuación se presenta la clasificación de esta patología basado en los genes involucrados de Tipo I a Tipo XV. 


\section{Osteogenesis imperfecta}

Vol. 3, núm. 1., (2019)

Alicia Cumanda Solano Barona; Deborah Denisse Jaramillo Cañadas; Karen Gissela Moreira Cusme; Karym Gabriela Jácome Vera

- Tipo I es la forma más leve de OI, presenta fracturas múltiples, esclerótica azul, quebradiza dientes, y pérdida de audición. Las fracturas son comunes en el período neonatal, pero raras en el útero o después de la edad adulta.

- Tipo II es el tipo más grave, con frecuencia causa la muerte al nacer o poco después porque ocasiona problemas respiratorios deformidad ósea severa, pobre mineralización, rebordeo de costillas, acortamiento de huesos largos y fracturas múltiples.

- Tipo III es el tipo más grave en los niños. La esclerótica azul es rara en este tipo, cara triangular, escoliosis (curvatura anormal de la columna vertebral) dientes frágiles, posible pérdida de audición y a menudo, severa deformidad ósea.

- Tipo IV similar al tipo 1 pero con deformidad ósea leve a moderada. Los pacientes no tienen esclerótica azul, cabeza grande, moretones fáciles y sin embargo el acortamiento de los huesos largos es más evidente después de que la edad adulta ha sido alcanzada.

\section{Clasificación expandida}

La clasificación genética molecular de OI ha demostrado ser muy heterogéneo, con diferentes y amplios patrones de herencia lo que genera variabilidad en la gravedad clínica.

Los investigadores (Marini JC, 2013) en su estudio notaron que:

Algunas personas que están clínicamente dentro del grupo tipo IV tenían un patrón distinto en sus huesos. Cuando revisaron la historia médica completa de estos pacientes, encontraron los grupos Tipo V y Tipo VI de OI. Las mutaciones causantes de estas formas de OI no se han identificado, pero las personas en estos dos grupos no tienen mutaciones en los genes de colágeno tipo 1 . 


\section{Osteogenesis imperfecta}

Vol. 3, núm. 1., (2019)

Alicia Cumanda Solano Barona; Deborah Denisse Jaramillo Cañadas; Karen Gissela Moreira Cusme; Karym Gabriela Jácome Vera

- El tipo V es similar a la OI tipo IV en apariencia y síntomas. La esclerótica es normal en color. Callos inusualmente grandes, llamados hipertrópicos Los callos se presentan en los sitios de fracturas o procedimientos quirúrgicos y se denominan "malla" debido a la apariencia del hueso cuando se observa bajo el microscopio.

- El tipo VI, es una forma autosómica recesiva de la enfermedad que puede ser causado por una mutación homocigótica en el gen SERPINF1 en el cromosoma 17p13.3, causando un defecto en la mineralización del cartílago. Presenta aspecto distintivo de "escala de pez" al observar el hueso bajo el microscopio. (M, 2013).

- Tipo VII, resultado de la herencia recesiva de una mutación en el gen CRTAP en homocigosidad o heterocigosidad compuesta en el cromosoma 3p22. La expresión parcial de CRTAP conduce a moderada displasia ósea, anomalías esqueléticas y huesos frágiles, existe entre 2 a 3\% de los casos de OI letal. (Barnes AM, 2006).

- Tipo VIII, deficiencia severa de crecimiento y esqueleto extremo bajo. mineralización. Es causada por ausencia o deficiencia severa de prolyl. Actividad de 3-hidroxilasa debido a mutaciones en el gen LEPRE 1 en el cromosoma 1p34.2 se asocia con OI grave o letal (Cabral WA, 2007).

- El tipo IX es una forma autosómica recesiva de OI que corresponde a tipos clínicamente graves II / III de la clasificación de silencio. Puede ser causado por una mutación homogénea en el gen PPIB en el cromosoma 15q22.31. No hay reportes de dentinogénesis imperfecta. (van Dijk FS, 2009)

- El tipo X es una forma autosómica recesiva de la enfermedad que puede ser causado por una mutación homocigótica en el gen SERPINH1 en cromosoma 11q13.5. Se 


\section{Osteogenesis imperfecta}

Vol. 3, núm. 1., (2019)

Alicia Cumanda Solano Barona; Deborah Denisse Jaramillo Cañadas; Karen Gissela Moreira Cusme; Karym Gabriela Jácome Vera

caracteriza por deformidades óseas y Fracturas múltiples, osteopenia generalizada, dentinogénesis imperfecta, y la esclerótica azul [16]. Tipos remanentes de genes de mutación de OI tipo XI. (Christiansen HE, 2010)

\section{Métodos de diagnostico}

A menudo es posible diagnosticar la OI basándose únicamente en las características clínicas. Los genetistas clínicos pueden realizar exámenes bioquímicos (colágeno) o moleculares con pruebas (de ADN) que pueden ayudar a confirmar un diagnóstico de OI en algunas situaciones, estas pruebas generalmente requieren varias semanas antes de que se conozcan los resultados.

En un estudio realizado por The National Institute of health (2012) "se cree que tanto la prueba de biopsia de colágeno como la prueba de ADN detectan casi el $90 \%$ de todas las mutaciones de colágeno tipo 1. El examen prenatal de rutina por ultrasonido o pruebas genéticas puede lograr buenos resultados para pacientes con antecedentes familiares positivos” (p. 23). Sin embargo, la tasa de detección es muy baja donde no hay antecedentes familiares, lo que puede dar lugar a diagnósticos erróneos, errores de diagnóstico o litigios. Los especialistas y médicos deben tener un alto índice de sospecha para tales casos. A continuación se definen los métodos empleados para diagnosticar esta enfermedad:

- Histomorfometría: En una pequeña minoría de casos la biopsia ósea muestra datos específicos anormalidades, que ayudan a establecer el diagnóstico de OI. Las biopsias deben ser de excelente calidad y debe ser examinado por observadores que están familiarizados con OI. (Glorieux F, 2009) 


\section{Osteogenesis imperfecta}

Vol. 3, núm. 1., (2019)

Alicia Cumanda Solano Barona; Deborah Denisse Jaramillo Cañadas; Karen Gissela Moreira Cusme; Karym Gabriela Jácome Vera

- Medición de densidad ósea (DMO): Aunque la DMO ahora está ampliamente disponible, pocos pacientes con OI tienen. En la mayoría de los casos, sin embargo, la DMO está muy por debajo del rango normal, así lo muestra la puntuación $\mathrm{Z}$ en niños y la puntuación T en adultos. La osteoporosis, la baja DMO es probablemente un factor de riesgo importante para mayor fracturas Sin embargo, la definición de osteoporosis desarrollada por la La Organización Mundial de la Salud, presenta una puntuación T inferior a 2,5 que no ha sido validado prospectivamente en adultos con OI. Por tanto en ninguna medida de DMO el uso de ultrasonido ha sido validado con OI. (Zionts LE, 2015).

- Ultrasonido: Siendo un método no invasivo, el ultrasonido es el principal método de selección prenatal. La mayoría de los casos de OI diagnosticados prenatalmente por ultrasonido son Tipo II y tipo III en menor escala. Esto se debe a que los tipos I y IV pueden ser normales antes del nacimiento, y los tipos V a VIII son extremadamente raros en práctica clínica. (Bulas D, 2010).

Las anomalías encontradas incluyen ecos reducidos, acortamiento de los huesos, angulaciones, cambios de curvatura, múltiples, fracturas y rebordes de las costillas. La discontinuidad del hueso también puede causar una apariencia arrugada. "La ecografía transvaginal puede detectar anormalidad en la semana 14, mientras que la ecografía transabdominal puede detectar anomalías solo después de las semanas 15 o 16" (Albin, 2007).

- Rotación de calcio, fosfato y hueso: El calcio sérico es normal en pacientes con OI la hipercalciuria se ha reportado en algunos pacientes con inmovilidad y con dificultades 


\section{Osteogenesis imperfecta}

Vol. 3, núm. 1., (2019)

Alicia Cumanda Solano Barona; Deborah Denisse Jaramillo Cañadas; Karen Gissela Moreira Cusme; Karym Gabriela Jácome Vera

renales o con nefrocalcinosis. La 25-hidroxi-vitamina D a menudo es baja, lo que indica una deficiencia de vitamina D por falta de exposición a la luz solar. (Glorieux F, 2009).

- Radiografía del útero: La radiografía de útero se puede utilizar cuando las pruebas de ecografía y la genética no han podido diagnosticar casos muy sospechosos. En conjunto con los cambios comunes de la OI, esta técnica puede detectar el hueso wormiano. Un cráneo único de hueso rodeado de suturas. Cuando el número de huesos es mayor que 10, esto se considera un número significativo de huesos wormianos (SNWB) y donde OI son altamente probables. (Shapiro JR, 2005).

En un estudio realizado por (Semler O, 2010) donde se utilizaron 195 casos de OI (tipo I, III y IV). Se encontró la incidencia de SNWB en porcentajes de 35\%, $96 \%$ y $78 \%$ en cada tipo respectivamente. Declararon que el SNWB tiende a aparecer en casos severos considerándose una herramienta confiable para el diagnóstico. Sin embargo, este método puede causar lesión por radiación.

Análisis de colágeno y diagnóstico genético: La biopsia de piel solía ser el principal método de diagnóstico de la OI. Sin embargo, este método demoraba mucho tiempo (varias semanas) y proporcionaba una mala exactitud.

Complicaciones de enfermedades de la salud relacionadas con la OI:

En pacientes con OI el mantenimiento de la salud es muy importante de lo contrario, las deformidades óseas y los defectos del colágeno comunes a la OI pueden afectar varios órganos internos, lo que lleva a problemas secundarios mayores o menores. 


\section{Osteogenesis imperfecta}

Vol. 3, núm. 1., (2019)

Alicia Cumanda Solano Barona; Deborah Denisse Jaramillo Cañadas; Karen Gissela Moreira Cusme; Karym Gabriela Jácome Vera

El autor (Forlino A, 2011) en su estudio expresa que las complicaciones que se presentan a raíz de la OI incluyen: Problemas pulmonares, problemas cardiacos, problemas neurológicos, coagulación sanguínea anormal, talasemia, periodontitis, función gástrica, cálculos renales, impresión basilar (BI), audición, visión, retraso en la sanación, masa ósea y deformidad ósea.

\section{Tratamiento}

Las fracturas deben tratarse lo más pronto posible al estar en presencia de una de ella con un control adecuado que requiere especialistas y médicos tratantes. Las terapias físicas deben ser personalizadas para cada paciente, a través de las terapias de rehabilitación se busca mantener la función lo más óptimo posible en todos los aspectos de vida del paciente. Se requiere de una adecuada instrucción y educación a los cuidadores, esto con la finalidad de disminuir al mínimo el riesgo de fractura. Se recomienda realizar ejercicios físicos en especial natación, ya que, aporta beneficios positivos en los pacientes, además se recomienda el uso de colchones especiales para facilitar la movilidad del niño en la cama.

El autor (Cassidy Suzanne B, 2005) en su estudio expresa que:

Las fracturas desplazadas deben ser cuidadosamente alineadas y enyesadas y, en caso necesario, se debe recurrir a la cirugía. Desde hace algún tiempo se están utilizando los Bifosfonatos para el tratamiento de la OI. Estos son compuestos sintéticos análogos de los pirofosfatos inorgánicos, utilizados con éxito en el tratamiento de la osteoporosis posmenopáusica y de la hipercalcemia del cáncer y actúan inhibiendo la reabsorción ósea por los osteoclastos. Su uso debe ser reservado para casos con síntomas severos en las OI tipo III y IV. Se ha reportado aumento de la densidad ósea, aumento del área vertebral, 


\section{Osteogenesis imperfecta}

Vol. 3, núm. 1., (2019)

Alicia Cumanda Solano Barona; Deborah Denisse Jaramillo Cañadas; Karen Gissela Moreira Cusme; Karym Gabriela Jácome Vera

disminución del número de fracturas y del dolor y mejoría en el manejo ambulatorio del paciente. (p. 56).

En la mayoría de los casos se usa pamidronato por vía endovenosa para tratar esta patología, sin embargo (Garcia, 2005) expone que "la eficacia del alendronato por vía oral es un excelente tratamiento que brinda mayor comodidad de administración y menos costos al paciente". A nivel neurológico se recomienda la realización de tomografías computarizadas de cráneo de control y de ser necesario el tratamiento quirúrgico.

La OI represente un grupo de enfermedades que puede ser en algunos casos letales y en otros invalidantes, por tal motivo es realmente importante tener un conocimiento acertado para poder generar un diagnóstico preciso que conlleve al manejo y tratamiento adecuado y que ayude a comprender el duro proceso que le toca vivir a quienes la padecen y a sus familiares.

\section{Discusión y conclusiones.}

La osteogénesis imperfecta es un trastorno heterogéneo con un amplio espectro de caracteres clínicos y una gran diversidad genética. A pesar de que la mayoría de los casos de OI son causados por mutaciones de COLIA1 / A2, estudios mencionado han revelado nuevas causas genéticas que han sido identificadas en los últimos años.

Algunos de estos son los genes que están relacionados con el procesamiento del colágeno tipo 1. En el presente estudio, el autor hace una descripción sistemática de la OI con características clínicas, clasificación y diagnóstico. La revisión también se centra en la OI relacionada con tantas 


\section{Osteogenesis imperfecta}

Vol. 3, núm. 1., (2019)

Alicia Cumanda Solano Barona; Deborah Denisse Jaramillo Cañadas; Karen Gissela Moreira Cusme; Karym Gabriela Jácome Vera

enfermedades de salud que crean preocupación en pacientes con esta patología y que incentivan campañas de mantenimiento de la salud.

Es muy importante tomar conciencia de la salud cuando se tiene presencia de esta enfermedad, ya que, de lo contrario, las deformidades óseas y defectos de colágeno común pueden afectar a varios órganos internos, trayendo como consecuencia problemas secundarios menores. Todavía no existe una cura para la OI, solo se emplea la administración de tratamiento para prevenir o controlar los síntomas que varían de persona a persona y que son expresados por el autor en el desarrollo del trabajo de investigación.

La terapia génica puede ser una posible opción de tratamiento futuro para la OI severa, sin embargo, los científicos siguen investigando un tratamiento prometedor para la OI.

\section{Bibliografía.}

Albin. (2007). Osteogenesis imperfecta: una revisión bibliografica.

Barnes AM, C. W. (2006). Deficiency of cartilage-associated protein in recessive lethal osteogenesis imperfecta. .

Biggin A, M. (2014). Osteogenesis imperfecta: diagnosis and treatment.Curr Osteoporos.

Bolívar, J. (2015). Investigación Documental. México. Pax.

Bulas D, S. H. (2010). Apariencia prenatal variable de osteogénesis imperfecta.

Cabral WA, C. W. (2007). Prolyl 3- hydroxylase 1 deficiency causes a recessive metabolic bone disorder resembling lethal/ severe osteogenesis imperfecta.

Cassidy Suzanne B, A. J. (2005). Management Of Genetic Syndromes.

Castro. (2016). Técnicas Documentales. Mexico: Limusa.

Castro, J. (2016). Técnicas Documentales. México. Limusa. 


\section{Osteogenesis imperfecta}

Vol. 3, núm. 1., (2019)

Alicia Cumanda Solano Barona; Deborah Denisse Jaramillo Cañadas; Karen Gissela Moreira Cusme; Karym Gabriela Jácome Vera

Christiansen HE, S. U. (2010). Homozygosity for a missense mutation in SERPINH1, which encodes the collagen chaperone protein HSP47, results in severe osteogenesis imperfecta. .

Dávila. (2015). Diccionario de Términos Científicos. Caracas: Oasis.

Forlino A, C. W. (2011). Nuevas perspectivas sobre la osteogénesis imperfecta.

Garcia, P. (2005). Curación ósea en niños con osteogénesis imperfecta tratados con bifosfonatos.

Glorieux F, B. N. (2009). Osteogenesis Imperfecta.

Glorieux FH, B. N. (2009). Cyclic administration of pamidronate in children with severe osteogenesis imperfecta. .

health, N. i. (2012). Osteoporosis and related bone diseases Osteogenesis Imperfecta overview. Obtenido de . www.bones.nih.gov.

Joseph L S, C. A. (2015). Desarrollo recienteen osteogénesis imperfecta.

M, B. (2013). Genotype-phenotype study in type V osteogenesis imperfecta. Clin Dysmorphol .

Marini JC. (2013). Nuevos genes en el desarrollo óseo: novedades en la osteogénesis imperfecta.

Rauch F, G. F. (2010). Osteogenesis imperfecta.

Salvador, L. (2011). Osteogénesis imperfecta Tipo I-IV, Trastorno del tejido conectivo .

Semler O, C. M. (2010). Wormian bones in osteogenesis imperfecta: Correlation to clinical findings and genotype. .

Shapiro JR, C. C. (2005). Efecto del pamidronato sobre el recambio óseo e IGF-I en la osteogénesis imperfecta tipo 1.

Sillence DO, S. A. (1979). Genetic heterogeneity in osteogenesis imperfecta. .

Starr, R. (2010). Osteogenesis imperfecta: primary care. Pediatr.

van Dijk FS, N. I. (2009). PPIB mutations cause severe osteogenesis imperfecta. .

Wagner, G. (2012). The status, quality, and expansion of the NIH full-length cDNA project: the Mammalian Gene Collection (MGC). Genome Res 14: 2121-2127.

Zhao X, Y. S. (2011). Recent progress in osteogenesis imperfecta. Orthop. 


\section{Osteogenesis imperfecta}

Vol. 3, núm. 1., (2019)

Alicia Cumanda Solano Barona; Deborah Denisse Jaramillo Cañadas; Karen Gissela Moreira Cusme; Karym Gabriela Jácome Vera

Zionts LE, N. J. (2015). Densidad mineral ósea en Niños con osteogénesis leve imperfecta. 